\title{
MATLAB Simulations based Identification Model for Various Points in Global Positioning System
}

\author{
Sekath Varma \\ Grenoble Ecol di Management \\ Business School \\ France
}

\author{
K. Nithiyananthan, PhD \\ Karpagam college of \\ Engineering \\ Coimbatore-641032 \\ Tamilnadu \\ India
}

\begin{abstract}
The main aim of this research work is to propose a probabilistic methodology to estimate the location of point $\mathrm{P}$ in global positioning system and to simulate it using MATLAB. The GPS receiver requires a minimum of four satellites to provide a three-dimensional (3D) fix and three satellites to provide a two-dimensional (2D) fix.A threedimensional (3D) fix means GPS can locate its latitude, longitude and altitude, while a two-dimensional (2D) fix means GPS locates only its latitude and longitude The focuses with "di" separations from every satellite $i$, characterizes a circle in the space accepting the earth to be round. The crossing point of these two circles (the earth and circle characterized by every one of the focuses with "di" separations from every satellite "i") is a circle on the world's surface. The circles shaped with a satellite and earth meet precisely at one point P. It is difficult to decide the "di" removes precisely, along these lines the circles don't meet at one accurate point. Every circle meets at two unique focuses. Subsequently, it has been recognized that the crossing point of the circles point $\mathrm{P}$ lies around there, however hard to find and distinguish the definite separation point $\mathrm{P}$. The proposed MATLAB simulation model has been able to locate various points in Global Positioning System accurately.
\end{abstract}

\section{Keywords}

GPS, MATLAB simulations, Receiver, Satellites, GPS control station, Trilateration.

\section{INTRODUCTION}

The Global Positioning System (GPS) is a space based radio positioning or navigation system that will provide threedimensional position, time and velocity information in any type of weather, anywhere on or near the Earth to suitably equipped users. The GPS program provides critical capabilities to military, civil and commercial users around the world. It is an engine of economic growth and jobs, and has generated billions of dollars of economic activity. It maintains future war-fighter advantage over opponents and is one of the four core military capabilities. In addition, GPS is the backbone for modernizing the global air traffic system. It is maintained by the United States government and is freely accessible by anyone with a GPS receiver with some technical limitations. GPS satellites orbit high enough to avoid the problems associated with land based systems, yet can provide accurate positioning 24 hours a day, anywhere in the world. Uncorrected positions determined from GPS satellite signals produce accuracy in the range of 50 to 100 meters[1]. When using a technique called differential correction, users can get positions accurate to within 5 meters or less[7]. The Global Positioning system comprises of three main segments. Each segment plays its own significant role in determining the position of the receiver and mapping locations of the receiver.
These segments are dependent on each other and are considered to be the most vital segments of GPS [2]. E

The three major segments are:

- $\quad$ GPS Satellites.

- GPS Ground Control Stations.

- $\quad$ GPS Receivers.

A GPS device determines its place by precisely transmitting the signals sent by GPS satellite high above the Earth. Each satellite regularly sends information that includes the time the concept was transmitted and precisely orbital details. The general system health and tough orbits of all GPS satellites are also transmitted [7]. The device uses the information it obtains to determine the transportation duration of each concept and determines the distance to each satellite. These ranges along with the satellite positions are used with the possible aid of trilateration, based on which criteria are used, to estimate the place of the device. This place is then viewed, perhaps with a moving map display or permission and longitude; level details may be included. Many GPS units show display details such as direction and rate, assessed for placement changes. Three satellites might seem enough to fix for a position since space has three dimensions and a place near the Global surface can be considered as well. However, even a very small clock error increased by the very huge rate of light (the rate at which satellite, alerts propagate) results in a huge positional error. Therefore, devices use four or more satellites to fix for the receiver's position and time. The very effectively calculated time is effectively invisible by most GPS applications, which use only the position [7]. A GPS receiver requires a minimum of three satellites to provide a 2-dimensional (2D) fix and four satellites to provide a 3-dimensional (2D) fix. A 2dimensional (2D) fix means that a GPS can locate its latitude and longitude while a 3-dimensional (3D) fix means that a GPS locates its latitude, longitude and altitude as well [3]. The process of determining relative or absolute locations of points by measurement of distances, using the geometry of circles, spheres or triangles are termed to be trilateration process. In three-dimensional geometry, it has been considered that a point lies on three sphere's surfaces and then the centers of the spheres along with the radii of the sphere provide information that helps to narrow down the probable locations of the point to be no more than two. If the point is known to be on the surface of a 4th sphere, then with the help of the center and radii of the 4th sphere, it could able to determine a unique location of the point [7]. The constraints involved in the Trilateration process to find the point of intersections with the help of geometry of circles, triangles and spheres [6] were explained.

For determination of the position of the receiver on the 
surface of the earth, it is required to calculate and compare the time signal that is sent to the receiver and that is received from the receiver by the satellite. From comparing both the time signals it is required to estimate the distance between them and by the process of trilateration and could able to estimate the probable position of the receiver. The problem lies in the determination of the exact runtime of signals. It has been known that all clocks of satellites are absolutely precise (they are atomic clocks after all) but the problem is the clock in GPS receiver. Atomic clocks being too expensive, GPS receivers are based on conventional quartz clocks which are comparatively inaccurate. Two satellites have been considered with the 1st satellite having its signal transmission time to be $5 \mathrm{Sec}$ and the 2nd satellite having its signal transmission time to be $4 \mathrm{Sec}$. In reality the actual time span for signals from the satellite to the receiver lies in the range of $0.07 \mathrm{~s}$ [7].

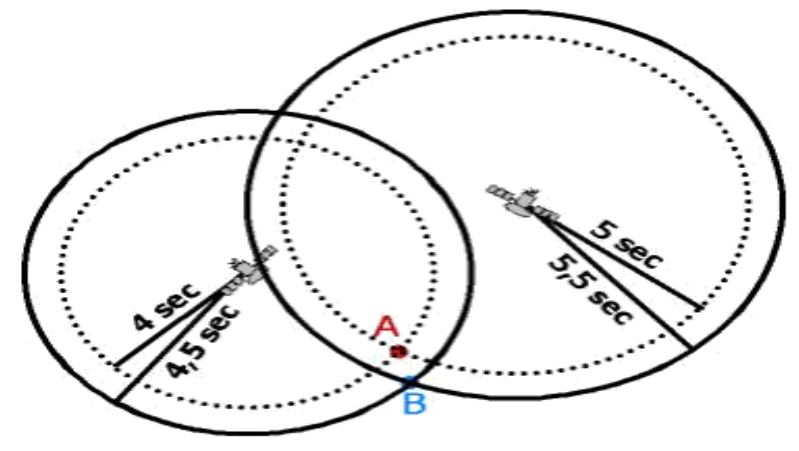

Figure 1 Two satellites transmission range

In Figure 1 it has not been considered the delay caused by the clock. It has been considered that the clock of the receiver to be 0.5 seconds.

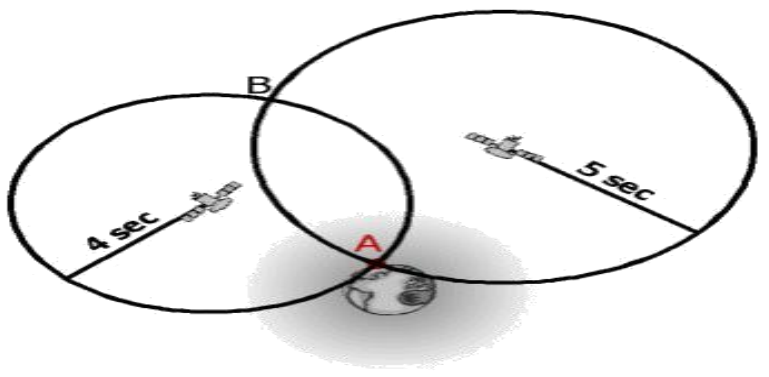

Figure 2 Two circles transmission range included with the Clock speed of the receiver

Figure 2 has two circles, one is the signal sent by the satellite that has accurate values which intersects at point $\mathrm{A}$ and the second circle seems to be the time the signal is sent by the receiver as it has clock to be added in addition to the signal which intersects at point $\mathrm{B}$. So the intersection point $\mathrm{B}$ can be determined as the pseudo ranges [4]. So with the increase of clock speed the pseudo range increases and vice versa. It needs to add another satellite, which has its signal transmission time to be 6 seconds[7].By adding the third satellite pseudo range had obtained as an area and the point A that is the intersection of the 3 circles without the delay are considered to be more accurate than the intersection of two circles as the receiver quartz clock is synchronized with the atomic clocks of the satellites and the GPS receiver also can be considered as an atomic clock. Now the pseudo ranges can be neglected and an accurate position can be considered [7].

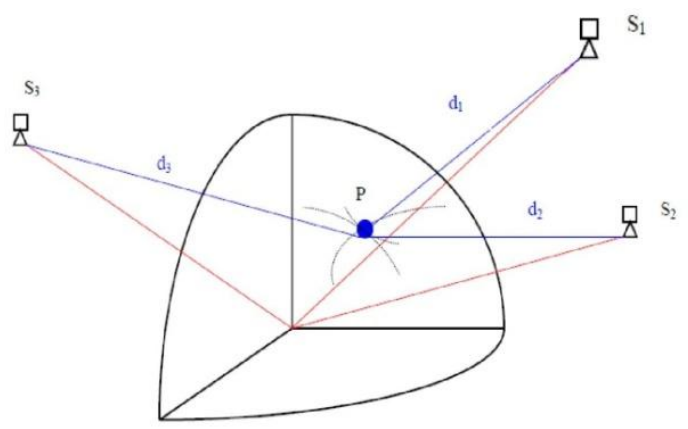

Figure 3 Distances from receiver to point $P$

From Figure 3 it has been clearly noticed that it is not possible to locate point $\mathrm{P}$ exactly because of an error factor, actually the point lies in an area created by the intersection of the three spheres. The area in which the point $\mathrm{P}$ lies is known as the ambiguity area as shown in Figure 4.

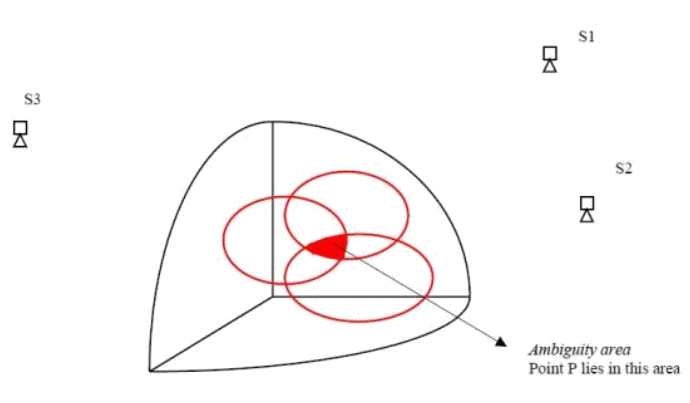

Figure 4 Location of $P$ in the ambiguity area

Figure 4 shows three circles which are the projections of the 3 satellites on a probable location. Their intersection area is considered to be the ambiguity area and the position of the receiver is considered to be located inside the ambiguity area. The MATLAB based simulations are carried out [5], which will take all the distances (distance between point and the satellites) sent by the satellites, longitude and latitude as inputs and try to simulate a probable location of point $\mathrm{P}$ into a 3-dimensional view of the using estimation of point using center of mass of probability and using most probable point[7].

\section{PROBABILSTIC SATELLITE DISTANCE CALCULATION MODEL}

In this research paper probabilistic satellite separation figuring model has been proposed. Through this scientific model point $\mathrm{P}$ in Figure 3 can be resolved precisely with a blunder variable. $\mathrm{P}$ is the area purpose of GPS yields. RE is the span of the earth E. ri is the separation of the satellite $\mathrm{i}$ from the focal point of the direction system.o is the focal point of the earth and the focal point of our direction system.di is the separation whenever send from the satellite to our point P.It is accepted that yield from the GPS recipient, dio is the information sent from GPS satellites.di is an irregular variable and $\mu=$ dio and $\sigma=50 \mathrm{~m}$. The client side of the GPS beneficiary needs the yield as sidelong and longitudinal directions on earth, the inputs of the collector will be sweep of the earth; $r=\operatorname{RE}$ (Assumption: Earth is thought to be round). Spot on satellite I (for me $=1 \ldots \mathrm{N}$ ); $\alpha \mathrm{i}, \beta \mathrm{i}$, ri in round directions. Separation between satellite $(i=1 \ldots N)$ and our area; dio. (Taken from satellite i, by the GPS gadget). When Three Spheres intersect:For the crossing point of three circles Trilateration strategy has been executed. The crossing points 
of the surfaces of three circles are found by defining the comparisons for the three circle surfaces and after that settling the three mathematical statements for the three questions, $\mathrm{x}, \mathrm{y}$, and z. To streamline the computations, the mathematical statements are defined so that the focuses of the circles are on the $\mathrm{z}=0$ plane. Likewise the plan is such that one focus is at the source, and each other is on the x-pivot. It is conceivable to define the mathematical statements in this way since any three non-direct focuses lie on a one of a kind plane[7]. Subsequent to finding the arrangement, it can be changed back to the first three dimensional Cartesian coordinate framework.

$$
\begin{aligned}
& r_{2}^{2}=(x-d)^{2}+y^{2}+z^{2} \\
& r_{3}^{2}=(x-i)^{2}+(y-j)^{2}+z^{2}
\end{aligned}
$$

It is needed to find a point located at $(\mathrm{x}, \mathrm{y}, \mathrm{z})$ that satisfies all three equations. Subtract the second equation from the first and solve for $\mathrm{x}$ :

$x=\frac{r_{1}^{2}-r_{2}^{2}+d^{2}}{2 d}$

It has been assumed that the first two spheres intersect in more than one point that is

$$
d-r_{1<} r_{2} d+r_{1}
$$

For this situation, substituting the comparison for $\mathrm{x}$ once again into the mathematical statement for the primary circle delivers the mathematical statement for a circle, the answer for the convergence of the initial two circles Substituting into the recipe for the third circle and illuminating for $y$ there results:

$$
y=\frac{r_{1}^{2}-r_{3}^{2}-x^{2}+(x-i)^{2}+j^{2}}{2 j}=\frac{r_{1}^{2}-r_{3}^{2}+i^{2}+j^{2}}{2 j}-\frac{i}{j} x
$$

$$
y^{2}+z^{2}=r_{1}^{2}-x^{2}
$$

The above $\mathrm{x}$ - and $\mathrm{y}$-coordinates of the solution point; it can simply rearrange the formula for the first sphere to find the $\mathrm{z}$ coordinate:

$$
z= \pm \sqrt{r_{1}^{2}-x^{2}-y^{2}}
$$

The answer for every one of the three focuses $\mathrm{x}, \mathrm{y}$ and $\mathrm{z}$. Since $\mathrm{z}$ is communicated as the positive or negative square root, it is workable for there to be zero, maybe a couple answers for the issue [7]. In the event that there are " $n$ " satellites, then the quantity of circles is likewise ' $n$ '. There are no (n-1)/2 crossing circle sets. There are (n-1) meeting focuses. Wipe out the focuses that don't need by computing the separation between the focus and the focal point of the circles. On the off chance that the separation is bigger than the distance across the circle dispose of the point, the blue spots in Figure 8. Therefore the limit of the point $\mathrm{P}$ has been recognized that the triangular shape limited by the red spots in Figure 8 . For each of the three red specks on the above figure, there are diverse $\alpha$ and $\beta$ 's (the sweep of the earth for every one of them are same not surprisingly). It has been discovered $\alpha \max , \alpha \min , \beta \max , \beta \min$ from these sets to make a rectangular region around point $\mathrm{P}$.

\section{RESULTS AND DISCUSSION}

In the proposed MATLAB based simulation model [8-9], it has been viewed as that the quantity of satellites I for finding the position $\mathrm{P}$ to be 5 to get the best plausible area. The Inputs that are to be entered are the scope of the point, the longitude of point and the quantity of satellites.Figure 6 demonstrates the evaluated three dimensional yield for the standard info parameters for the proposed model.

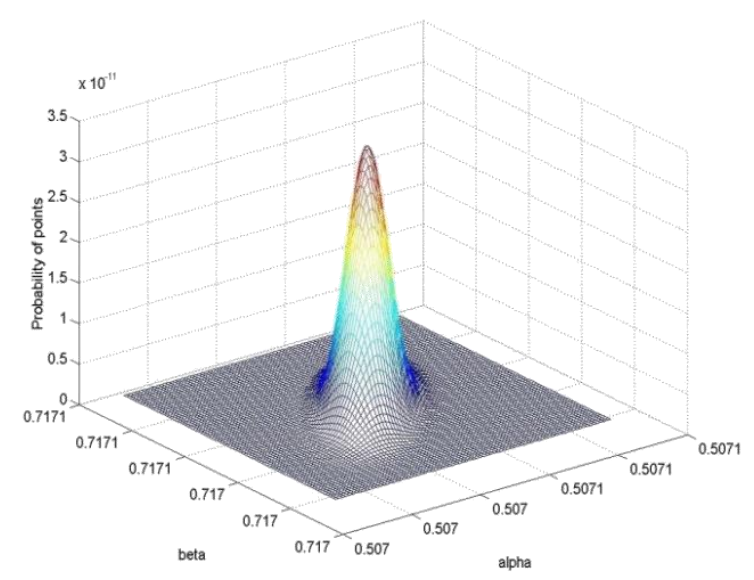

Figure 6 The final estimated 3-D output of the proposed model

It clearly indicates the alpha and beta are on the $\mathrm{x}$ and $\mathrm{z}$ axis respectively. Probability of points is indicated on the $\mathrm{Y}$ axis. The above diagram is a $3 \mathrm{D}$ view of the probable position of the receiver which includes the altitude the longitude and the latitude of the receiver on the surface of the earth [7].

\section{CONCLUSION}

In the proposed model with the help of a probabilistic approach an attempt has been made to estimate the position and the error of the GPS receivers. A mathematical model has been constructed and simulation has been implemented in order to get the accurate position and error of the GPS receivers. In this research work, it has been observed that the location and error of the receivers depend on sigma of normal probability distribution function of the distance between the point and satellites when different sigma values are in use. The estimation of the location of the probable point would be similar but the error would change with respect to the altering sigma values [8]. Hence it is very hard to find, out the ambiguity area by using the only mathematical model equations and calculate it. This constraint has been taken into consideration and the concept of Differential Global Positioning System is used as in the reference station is added to the proposed GPS simulation which increases the accuracy of the location of the receiver. 


\section{REFERENCES}

[1] J. Thor and D.M. Akos. A direct RF sampling multifrequency GPS receiver. Proc. IEEE Position Location and Navigation Symp. Apr. 2002, pp. 44-51.

[2] Jiyu Liu. GPS satellite navigation and positioning theory and methods, Beijing. Science Press, 2003,118-152.

[3] Michael S. Bmsch. GPS Receiver Architectures and Measurements. Proc. of the IEEE, p.48-84, Vo1.87, No.1, January 1999.

[4] A. J. Van Dierendonck. GPS receiver in Global Positioning System: Theory and Application. Vol.1, B. W. Parkinson and J. J. Spilker, Jr., Eds. Washington, DC: American Institute of Aeronautics and Astronautics, 1996, ch. 8, pp. 329-407.

[5] J.B.-Y. Tsui. Fundamentals of global positioning system receivers. New York: Wiley, 2000.
[6] M. Jian, W. Chunli, L. Qiqin. Research on GPS simulation technique. IEEE Transactions on. Microwave and Millimeter Wave Technology, Apr. 2002, pp365368 .

[7] Sekethverma, K.Nithiyananthan 'Matlab Based Global Positioning System Simulations', Unpublished Thesis ,BITS Pilani - Dubai, 2012.

[8] S. Samson Raja, R. Sundar, T. Ranganathan, K. Nithiyananthan.K, "LabVIEW based Simple Load Flow Calculator Model for Three Phase Power System Network", International Journal of Computer Applications, Vol-132, Issue-2, 2015,9-12.

[9] Nithiyananthan.K and Ashish Kumar Loomba (2011) 'MATLAB/SIMULINK based Speed control model for converter controlled DC drives' International Journal of Engineering Modelling, Croatia, EUROPE, Vol. 24, No 1-4, pp.49-55. 